\title{
Construction Grammar meets Phraseology: \\ eine Standortbestimmung
}

Alexander Ziem (Düsseldorf)

\begin{abstract}
In the $1980 \mathrm{~s}$, the very idea of a constructionist approach to grammar was considerably motivated by the observation that irregular syntactic structures and semantic idiomaticity constitute a major part of a language. Even though, since then, a multitude of case studies followed the methodological spirit to approach fully regular "core" structures from insights into irregular structures (and not the other way around), it seems as if the genuine interest in idiomaticity has been decreasing in the last decade or so. At least it is fair to say that phraseology and construction grammar evolved apart from each other, ignoring largely how much they could profit from one another. This is even more surprising, since idiomaticity is arguably a driving force in the language system. Assuming that idiomaticity is at the heart of language, this article introduces the special issue "Construction Grammar meets Phraseology" by providing an overview of relevant concepts and research perspectives guiding the contributions to this issue. Featuring five constructionist case studies on phraseme constructions and partially filled argument structure constructions, the special issue not only subsumes in-depth analyses of a variety of constructional idioms within the lexicon-grammar continuum, but also makes the case for closer collaborations between construction grammar and phraseology both in terms of methodology and the linguistic phenomena addressed.
\end{abstract}

\section{Irregularitäten als grammatischer Gegenstandsbereich: Ausgangspunkte des Themenheftes}

Die Konstruktionsgrammatik - als eine alternative Grammatiktheorie - ist seit ihren Anfängen in den 1980er Jahren durch die Beobachtung motiviert, dass sich eine Fülle durchaus unterschiedlicher sprachlicher Phänomene nicht oder nur sehr unzureichend mithilfe regelbasierter Mechanismen erfassen und erklären lassen. In diesem Sinne ist es nicht übertrieben zu behaupten, dass Phraseme die vielleicht wichtigste treibende Kraft für die Konstruktionsgrammatik waren (cf. Fillmore/Kay/O'Connor 1988; Fillmore 1988; zusammenfassend auch Gries 2008: 12-15). Dies gilt bis heute, denn immer noch bilden idiomatische Einheiten den wohl größten Gegenstandsbereich konstruktionsgrammatischer Studien (cf. Taylor 2012: 71), auch wenn Michaelis (2012) zu Recht betont, dass sich transparente und völlig reguläre Strukturen keinesfalls eines konstruktionsgrammatischen Zugriffs entziehen. 
Der Mehrwert eines konstruktionsgrammatischen Ansatzes besteht darin, den großen Bereich des Idiomatischen, also semantisch und/oder syntaktisch Irregulären, nicht ohne Vorbehalt an das Lexikon zu delegieren und ihn mithin zu ignorieren, sondern ihn vielmehr als Prüfstein für die Adäquatheit einer Grammatiktheorie zu betrachten. Den Bereich des Irregulären ernst zu nehmen, ist dabei keine selbst auferlegte Beschäftigungstherapie der Konstruktionsgrammatik, sondern vielmehr eine Notwendigkeit, die sich aus dem Gegenstandsbereich selbst ergibt, denn viele irreguläre Strukturen können aufgrund ihres syntagmatisch komplexen und schematischen Charakters gar nicht ohne Weiteres als Einheiten des Lexikons behandelt werden. Leitend ist demnach der Anspruch, dass eine Grammatiktheorie in der Lage sein muss, auch nichtkompositionelle Einheiten erklären zu können, sie also nicht zu bloßen Ausnahmen zu degradieren. Dieser Anspruch lässt sich nur einlösen, indem an den irregulären Fällen aufgezeigt wird, welchen syntaktischen, semantischen und pragmatischen Beschränkungen ihre Realisierung gehorchen muss. Das Ziel muss somit darin bestehen, die Mechanismen der Erzeugung von neuen lizenzierten Instanzen vollständig in ein grammatisches Beschreibungsmodell zu integrieren.

Diesem Kerngedanken Rechnung tragend, war es Charles Fillmore, der als Erster den wegweisenden strategischen Vorschlag gemacht hat, den Bereich des Irregulären zum Ausgangspunkt einer Grammatiktheorie zu machen, ,specifically because we believe that insights into the mechanics of the grammar as a whole can brought out most clearly by the work of factoring out the constituent elements of the most complex constructions" (Fillmore 1988: 36). Im Anschluss an Fillmores Pionierarbeit - freilich unterstützt von zahlreichen KollegInnen wie Paul Kay, George Lakoff und Ronald Langacker - hat sich allmählich seit den 1980er Jahren eine scientific community herausgebildet, die trotz Unterschiede im Detail die Grundüberzeugung teilt, Konstruktionen, also Form-Bedeutungspaare variierenden Abstraktionsgrades, als grundlegende Bausteine einer Sprache anzusehen (cf. Ziem/Lasch 2013: Kapitel 5), die im breit aufgespannten Kontinuum zwischen idiomatischen Strukturen einerseits und regulären Mustern bzw. Regeln andererseits formal einheitlich zu beschreiben sind. Kay (2002: 1) begreift in diesem Sinne formal einheitliche Beschreibungen als eine Leitmaxime der konstruktionsgrammatischen Forschung: Es bestünde die Notwendigkeit, „to develop a system of grammatical description in which the marked constructions (more or less ,idiom-like' forms of expression) are represented in the same formal system as the regular ,core' patterns or rules.“

Knapp drei Jahrzehnte nach Fillmores weitsichtiger Vision sind wir zwar immer noch weit davon entfernt, die Mehrzahl der Konstruktionen, die die Grammatik einer Sprache konstituiert, identifiziert und deskriptiv erfasst $\mathrm{zu}$ haben, jedoch hat sich eine international wachsende Forschungsgemeinschaft gebildet, die sich genau dieser Aufgabe widmet (cf. den Überblick in Hoffmann/Trousdale 2013; für das Deutsche auch Ziem/Lasch 2013: 153-164). Leitend war und ist dabei die Maßgabe, im Rahmen eines konstruktionsbasierten Frameworks möglichst viele - idealiter alle - sprachlichen Phänomene einheitlich und konsistent zu beschreiben. Aus diesem Grund sprechen Fried und Östman (2004: 24) auch von einem maximalistischen Ansatz. Für die Analyse von Phraseoschablonen - und für schematische Einheiten generell - hat es sich als notwendig erwiesen, den Begriff der Konstruktion nicht auf (semantisch und / oder syntaktisch) idiomatische Einheiten zu reduzieren, sondern vielmehr auch solche sprachlichen Größen einzubeziehen, die zwar vollständig regulär, aber zugleich so rekurrent auftreten, dass

ISSN 1615-3014 
sie sich zu einer (mehr oder weniger) festen Mehrworteinheit konsolidiert haben. Diesem gebrauchsbedingten Umstand der Musterbildung trägt Goldberg in ihrer erweiterten Definition von Konstruktionen Rechnung, wenn sie schreibt: „In addition, patterns are stored as constructions even if they are fully predictable as long as they occur with sufficient frequency." (Goldberg 2006:5) Sprachgebrauchsbedingte Verfestigungsprozesse können sprachliche Strukturen verschiedener Komplexität betreffen, durchaus auch ganze Sätze (etwa im Fall von Sprichwörtern) und satzwertige Ausdrücke. Prototypische Vertreter sind aber eher kleinere Einheiten, so etwa schematische Mehrworteinheiten wie Phraseoschablonen (cf. Abschnitt 2) und grammatische Phraseme (wie geschweige denn), aber auch Kookkurrenzen (blöder Zufall, schöne Scheiße, Schulter an Schulter) und Begrüßungs- oder Abschiedsformeln (auf Wiedersehen, viele Grüße). Obwohl der Grad an Fixiertheit der lexikalischen Bestandteile variieren kann (cf. Schulter an Schulter vs. Auto an Auto, hierzu ausführlich: Abschnitt 2.2, Ziem im Druck), bleiben die komplexen Einheiten als Ganzheiten abrufbar. Es bedarf also keiner kompositionellen Zerlegung in (semantische und/oder formale) Bestandteile, um die Ausdrücke angemessen verstehen oder verwenden zu können.

Das vorliegende Themenheft knüpft an zentrale Fragestellungen im Spannungsfeld von Konstruktionsgrammatik und Phraseologie an. Ausgangspunkt des Themenheftes sind zwei Eigenschaften von Phrasemen und sprachlichen Mustern (cf. auch Ziem/Boas 2017): (a) Die in den Beiträgen des Themenheftes im Fokus stehenden Mehrworteinheiten sind im Übergangsbereich zwischen Lexikon und Grammatik zu verorten und können - müssen aber nicht - idiomatischer Natur sein; (b) sie lassen sich hinsichtlich ihrer Produktivität, Schematizität und jener Beschränkungen (constraints) untersuchen, denen sowohl die Füllung ihrer Leerstellen (insofern solche vorhanden sind) als auch ihre Verwendung (etwa hinsichtlich Register, Textsortenspezifik, Illokution der Äußerungseinheit etc.) unterworfen sind.

Darüber hinaus werden in dem Themenheft aber auch ganz grundsätzliche Fragestellungen berührt, die sich aus dem Verständnis von Konstruktionen als konventionalisierte FormBedeutungspaare ableiten. So ist vielen empirischen Herausforderungen bislang erstaunlicherweise nur wenig Beachtung geschenkt wurde, beispielsweise der Frage, (a) ob jedes Phrasem als eine Konstruktion anzusehen ist, (b) wann überhaupt eine rekurrente Mehrworteinheit als eine Konstruktion zu klassifizieren ist, (c) wie sich zwischen verschiedenen Verfestigungs- und Konventionalisierungsgraden von Konstruktionen unterscheiden lässt und (d) dabei der Konstruktionsbegriff sinnvoll in ein phraseologisches Beschreibungsmodell integriert werden kann (cf. hierzu Feilke 2007).

Das vorliegende Themenheft macht sich zur Aufgabe, den Dialog zwischen Konstruktionsgrammatik und Phraseologie unter den skizzierten Voraussetzungen zu beleben und $\mathrm{zu}$ vertiefen. Gegenstand des Themenheftes sind phrasemhafte Konstruktionen (im technischen Sinne der Konstruktionsgrammatik) ganz unterschiedlicher Ausprägung: von musterhaften teilspezifizierten Argumentstrukturen bis zu Somatismen. Das Themenheft setzt sich zum Ziel, Affinitäten und Diskrepanzen zwischen einem phraseologischen und konstruktionsgrammatischen Zugang vor dem Hintergrund kritisch zu reflektieren, dass sich insbesondere die Konstruktionsgrammatik in den letzten zwei Dekaden erheblich ausdifferenziert hat und es infolgedessen eine Fülle an fruchtbaren Anschlussstellen zur Phraseologieforschung zu geben scheint. 
Im Folgenden möchte ich zunächst einleitend das produktive Spannungsfeld zwischen Phraseologie und Konstruktionsgrammatik ausloten. Zuerst widme ich mich so genannten Phraseoschablonen; diese Untergruppe von Phrasemen bildet zweifelsohne den prototypischen Gegenstandsbereich konstruktionsgrammatisch orientierter Analysen in der Phraseologie (Abschnitt 2). Ein besonderes Augenmerk soll dabei auf konstruktionsgrammatisch relevanten Untersuchungsgesichtspunkten liegen, weil auch in diesem Themenheft Phraseoschablonen einen zentralen Untersuchungsgegenstand bilden. Gleichsam aus der Vogelperspektive werfe ich im Anschluss daran einen eher kursorischen Blick auf Forschungsschwerpunkte und Forschungsperspektiven im Schnittbereich von Phraseologie und Konstruktionsgrammatik (Abschnitt 3), bevor zu guter Letzt die einzelnen Beiträge des Themenheftes und deren inhaltliche Schwerpunktsetzungen kurz vorgestellt werden (Abschnitt 4).

\section{Das konstruktionsgrammatische Interesse an Phrasemen und das phraseologische Interesse an Konstruktionen}

\subsection{Phraseoschablonen als konstruktionsgrammatischer Gegenstandsbereich}

Schon ein flüchtiger Blick auf die einschlägige Fachliteratur zeigt, dass Phraseoschablonen als ein geradezu idealtypisches Untersuchungsgebiet der Konstruktionsgrammatik anzusehen sind (cf. hierzu ausführlich Dobrovol'skij 2011; auch: Bücker 2012; Feilke 2007; Gries 2008: 12-15; Mellado/Mollica/Schafroth im Druck; Staffeldt 2018: Kap. 1.8). Dies liegt nicht zuletzt daran, dass Phraseoschablonen - mitunter auch Konstruktionsidiome (cf. Booij 2002; Taylor 2012) oder Phrasemkonstruktionen (Dobrovol'skij 2011: 114) genannt - anders als andere Phraseme Leerstellen aufweisen. Unter einer Phraseoschablone wird gemeinhin eine schematische, syntagmatisch komplexe, mithin mehrgliedrige Einheit mit einer (mehr oder weniger) festen, d. h. nicht-kompositionellen, Bedeutung verstanden (cf. etwa Fleischer 1997: 131). Von anderen Phraseologismen unterscheiden sich Phraseoschablonen dadurch, dass „bestimmte Positionen in ihrer syntaktischen Struktur lexikalisch besetzt sind, während andere Slots darstellen, die gefüllt werden müssen“ (Dobrovol'skij 2011: 114). Diese inhärente Schematizität stellt hohe Anforderungen an die (semantische und syntaktische) Beschreibung von Phraseoschablonen, denn einerseits können sie nicht wie Lexeme behandelt werden, da sie nicht vollständig spezifiziert sind und die Lücken nicht frei gefüllt werden können, andererseits sind Phraseoschablonen aber ebenso wenig der Grammatik zuzuordnen, da es sich nicht um rein strukturelle Größen, sondern vielmehr um lexikalisch immer schon teilspezifizierte Einheiten handelt. Da Phraseoschablonen mithin im Übergangsbereich zwischen Lexikon und Grammatik angesiedelt sind, sind sie bislang kaum systematisch analysiert worden: Während sie aufgrund ihrer Schematizität zunächst gar nicht, seit einiger Zeit eher am Rande als phraseologisch interessante Phänomene wahrgenommen wurden, geraten sie schon deswegen kaum in den Blick der (traditionellen) Grammatikschreibung, weil sie sich in Absehung von ihren lexikalischen Bestandteilen nicht beschreiben lassen.

Phraseoschablonen treten im Deutschen in vielfältiger Form auf; sie sind phraseologisch gut dokumentiert (so etwa in Fleischer 1997), bis heute aber bei Weitem noch nicht vollständig erfasst (cf. aber die Pionierstudien zu usuellen Wortverbindungen in Steyer 2013). Phraseoschablonen weisen interne und/oder externe Leerstellen auf (cf. etwa je..., desto ..., Was für ein... vs. ganz gleich..., wie dem auch sei...); sie können funktional lexikalischen Kategorien 
ähneln (wie etwa der Konnektor geschweige denn), phrasaler Natur sein (cf. Arm in Arm, Frau von Größe) oder auch als satzwertige Ausdrücke auftreten (cf. Ich und Golfspielen, Du hast gut reden). Neben eigenständigen (oft schematischen) Bedeutungen können sie auch pragmatische Informationen transportieren, so etwa Einstellungen markieren (Lass...mal... sein) oder diskursfunktionale Aufgaben übernehmen (wie ...hin, ...her).

Der wohl wichtigste Unterschied eines phraseologischen und konstruktionsgrammatischen Zugangs zu Phraseoschablonen besteht darin, dass ersterer sich primär auf Aspekte der systematischen Klassifizierung und Dokumentation von Ausprägungsvarianten richtet, ${ }^{1}$ während letzterer den analytischen Fokus insbesondere auf strukturelle, semantische und pragmatische Beschränkungen der Realisierbarkeit einzelner Phraseoschablonen lenken (cf. etwa Jackendoff 2008; fürs Deutsche Finkbeiner 2017; Ziem im Druck). Ungeachtet dieser augenfälligen Divergenz bleibt jedoch festzuhalten, dass sich beide Zugänge gegenseitig insofern ergänzen und mithin aufeinander zubewegen, als es der Konstruktionsgrammatik darauf ankommt darzulegen, wie stark jeweils konstruktionsspezifischen Beschränkungen Rechnung zu tragen ist, wie sehr sich also Konstruktionen eines rein regelbasierten Zugriffs entziehen, während umgekehrt die Phraseologie zunehmend feststellen muss, dass es bei der Realisierung von phraseologischen Einheiten vielfach einen auszutarierenden Spielraum von Varianz gibt (cf. hierzu ausführlich Staffeldt 2018: Kap. 1.8.1). Phraseoschablonen bilden infolgedessen geradezu prototypische Einheiten im Übergangsbereich zwischen Grammatik und Lexikon, weil an ihnen deutlich wird, dass Wörter (und feste Wortverbindungen) einerseits und syntaktische Strukturen andererseits nicht kategorial verschieden sind. Sie sind weder als Einheiten des Lexikons, also als Gegenstand semantischer Analysen, noch als grammatische Größen, also als Gegenstand syntaktischer und morphologischer Analysen, zu behandeln, sondern zeichnen sich durch grammatische und lexikalische Eigenschaften aus.

Auch wenn phraseologische Konzepte und Ideen die Konstruktionsgrammatik seit ihren Anfängen begleiten, gibt es noch eine Fülle an offenen Forschungsfragen, so etwa die folgenden:

- Zeichentheoretisch entspricht der Übergang vom Konstrukt zur Konstruktion dem Wandel eines Zeichen-Tokens zu einem Zeichen-Type, wann aber ist dieser Prozess abgeschlossen? Wie lassen sich Prozesse und Grade der Verfestigung messen und beschreiben? Lassen sich diese Fragen ohne groß angelegte diachronische Untersuchungen beantworten (siehe Hoffmann/Trousdale 2011 fürs Englische und insbesondere Colleman/De Clerck 2011)?

- Welchen Kriterien muss eine phraseologische Einheit genügen, um den Status einer Konstruktion zu erlangen (und umgekehrt)? Anders formuliert: Wie lässt sich empirisch bestimmen, ob in einer Sprachgemeinschaft eine Konstruktion etabliert ist?

- Welche Bedingungen müssen erfüllt sein, um Konstrukte (Tokens) nicht mehr als Instanzen einer schon bestehenden Konstruktion (eines Types) anzusehen, sondern als Ausprägungen einer neuen Konstruktion, die auf einer höheren oder niedrigeren Ebene der Granularität angesiedelt ist?

\footnotetext{
${ }^{1}$ Zum Verhältnis von Lexikographie und Phraseologie unter Einbezug konstruktionsgrammatischer Aspekte cf. exemplarisch Dobrovol'skij 2010.
} 
- Insofern es sich bei konstruktionalen Einheiten um phraseologisch verfestigte Einheiten handelt, kommt ihnen der Status von Mustern mit herausgebildeten Standardwerten zu (im Sinne der Frame-Semantik, cf. Ziem 2008: 335-366), aber inwiefern können Methoden der quantitativen Korpusanalyse sowie stärker qualitativ ausgerichtete Zugänge (einschließlich der Frame-Semantik) dabei helfen, Konstruktionen verschiedenen Abstraktionsgrades zu erfassen und zu beschreiben?

- Manche strukturell-funktionalen Verfestigungen dienen als Muster, um bestimmte kommunikative Aufgaben zu erfüllen - wie lassen sie sich diese identifizieren, begründen und beschreiben?

- Lässt sich der Muster-Begriff jenseits der Satzebene auf die Text- und Diskursebene anwenden? Wenn ja, wie? Gibt es so etwas wie Textkonstruktionen und Diskurskonstruktionen (cf. hierzu Östman 2004)? Wie ließen sich solche Einheiten von Schreib- und Textroutinen abgrenzen (Feilke/Lehnen 2012)?

- Inwiefern, nach welchen Kriterien und mit welchen analytischen Mitteln können lexikalische Kookkurrenzphänomene, die eindeutig nicht in die Phraseologie gehören, jedoch nicht ganz vorhersehbar sind, als Konstruktionen betrachtet und behandelt werden?

- Gibt es bei lexikalisch nicht fixierten, d. h. semantisch-strukturellen Ebenen der Konstruktionsgrammatik, die außerhalb der Phraseologie $\mathrm{zu}$ liegen scheinen, Berührungspunkte mit dieser? Weisen z. B. caused motion-Konstruktionen oder Resultativkonstruktionen zumindest teilweise semantische oder lexikalische Regularitäten auf (cf. Dalmas/Gautier in diesem Band), durch die sie in die Nähe zu Phrasemen rücken?

Nicht alle diese Fragenkomplexe können freilich in den Beiträgen dieses Themenheftes in der gebotenen Ausführlichkeit behandelt und diskutiert werden, sie geben aber eine Richtung vor, in die sich die Zusammenarbeit zwischen Konstruktionsgrammatik und Phraseologie in der Zukunft entwickeln könnte. Unabhängig davon bleibt festzuhalten: So sehr sich das syntaktisch orientierte Interesse der Konstruktionsgrammatik an Phraseologismen von dem klassifikatorischen Interesse der Phraseologie unterscheidet, so sehr sind beide Zugänge gleichermaßen dadurch motiviert, das Reguläre im Irregulären zu erfassen. Dies kann jeweils nur gelingen, wenn die Varianz an Ausprägungsformen von Phraseoschablonen in den Blick genommen wird und dabei die (semantischen, syntaktischen, pragmatischen) Unterschiede herausgearbeitet werden sowie jene Vererbungsbeziehungen form- und inhaltsseitig modelliert werden, die eine Konstruktion $\mathrm{zu}$ anderen Konstruktionen innerhalb und jenseits ihrer Konstruktionsfamilie unterhält.

\subsection{Untersuchungsgesichtspunkte von Phraseoschablonen (und anderen Phrasem- Konstruktionen)}

Das konstruktionsgrammatische Interesse an Phrasemen ist immer dann besonders groß, wenn Mehrworteinheiten interne oder externe Leerstellen aufweisen. Bei solchen Phrasemen ist zugleich aus phraseologischer Perspektive der Bedarf an einer konstruktionsgrammatisch motivierten Beschreibung besonders stark. Relevant sind insbesondere folgende Untersuchungsgesichtspunkte: Musterhaftigkeit, Konstruktionsbedeutungen, Constraints, (typische) Filler der Leerstellen, Produktivität sowie (Vererbungs-)Beziehungen zu anderen 
Konstruktionen. Diese Gesichtspunkte sollen am Beispiel von nominalen Reduplikationskonstruktionen (cf. hierzu ausführlich Ziem im Druck) kurz vorgestellt werden.

Musterhaftigkeit. Musterhaft können Phraseoschablonen in mehrerlei Hinsicht sein. Zunächst bildet die jeweils syntagmatisch fixierte Abfolge von Konstituenten ein festes Muster. Die Konstituenten können dabei entweder lexikalisch spezifiziert sein oder unspezifiziert bleiben; im letztgenannten Fall handelt es sich um Leerstellen, die unter bestimmten Bedingungen ,gefüllt', also durch sprachliche Einheiten besetzt werden können. So zeichnet sich beispielsweise die Reduplikationskonstruktion mit der Präposition an (z. B. [Tür] an [Tür], [Auto] an [Auto]) durch die musterhafte Form $\left[\mathrm{NP}_{1}\right.$ an $\left.\mathrm{NP}_{1}\right]$ aus, wobei $\mathrm{NP}$ für „Nominalphrase“ steht und der Index „1“ anzeigt, dass es sich um dieselbe Nominalphrase handelt. Die Konstruktion besteht also aus der Kombination von drei Konstituenten, nämlich der Präposition an und einem artikellosen Nomen (ggf. als Kopf einer NP), das einmal linksund einmal rechtsadjazent zur Präposition realisiert wird. Weiterhin kann eine Konstruktion auch dann musterhaft sein, wenn ihre Leerstelle(n) sprachlich realisiert ist bzw. sind; dies ist dann der Fall, wenn die so realisierte Konstruktion eine eigene, feste bzw. verfestigte Bedeutung hat. Meist handelt es sich dabei um eine idiomatische Bedeutung. Bei der Reduplikationsstruktur mit an sind etwa die Nomen Tür und Schulter Kandidaten dafür, denn die Mehrworteinheit Tür an Tür auch die idiomatische Bedeutung ,ganz in der Nähe von“, , angrenzend', genauso wie die Mehrworteinheit Schulter an Schulter auch die idiomatische Bedeutung ,zusammen', ,koordiniert', eng miteinander abgestimmt' haben kann. ${ }^{2}$

Konstruktionsbedeutungen. Auch schematische Konstruktionen tragen Bedeutung oder erfüllen sprachliche Funktionen. Anders als lexikalische Einheiten sind ihre Bedeutungen jedoch oftmals schematisch und abstrakt. Das trifft auch auf Phraseoschablonen/konstruktionale Idiome zu. Eine zentrale Aufgabe konstruktionsgrammatisch orientierter Studien besteht darin, die Bedeutung oder Funktion einer Phraseoschablone derart zu bestimmen, dass der Beitrag ihrer (lexikalisch fixierten und variablen) Konstituenten zur Gesamtbedeutung bzw. -funktion deutlich wird. Die Reduplikationsstruktur $\left[\mathrm{NP}_{1}\right.$ an $\left.\mathrm{NP}_{1}\right]$ ist beispielsweise ambigue: Abhängig vom Nomen, das die Leerstellen besetzt, kann die Reduplikationsstruktur entweder eine kontinuative Lesart haben oder eine (ggf. metaphorisch verstandene) Nähe ausdrücken; ersteres exemplifiziert Auto an Auto (im Sinne von, ein Auto reiht sich an dem anderen'), letztgenannte Lesart liegt vor bei Tür an Tür (im Sinne von, direkt nebenan').

Typische Filler der Leerstellen. Die lizenzierten Filler einer Leerstelle können sich hinsichtlich ihrer Prototypikalität unterscheiden. So gibt es vielfach typische, d. h. eher erwartbare und frequenter realisierte Filler. Im Fall von Reduplikationsstrukturen mit an treten beispielsweise die Filler Tür und Hand sehr viel häufiger auf (zur quantitativen Erhebung von Belegstellen mit dem Tool „lexpan“ cf. Ziem im Druck). Zumindest bei Phraseoschablonen scheint es dabei einen direkten Zusammenhang zwischen Auftretensfrequenz der Filler und dem Grad an Idiomatizität zu geben: Je frequenter der Filler, desto höher sein Verfestigungsgrad. Die Auftretensfrequenz und der Grad an Verfestigung ist bei dem Nomen

\footnotetext{
${ }^{2}$ Cf. etwa folgende Belegstellen (aus dem DWDS-Kernkorpus 21): Und man möchte denken, daß die Feinde von gestern, sobald das Leben sich normalisiert, in Zukunft Schulter an Schulter arbeiten werden [...]. In diesem edlen Viertel logiert Karlheinz Schreiber Tür an Tür mit diplomatischen Eminenzen [...].
} 
Hand mit Abstand am größten; es ist kein Zufall, dass damit ein vergleichbar hoher Grad an Idiomatizität einhergeht, wenngleich die Bedeutung der Konstruktion semantisch transparent bleibt: Hand in Hand wird sowohl in der nicht-idiomatischen als auch in der idiomatischen Lesart verwendet (cf. Sie gingen Hand in Hand über die Straße vs. Die Ausbreitung der Krankheit geht Hand in Hand mit der demographischen Entwicklung).

Constraints. Konstruktionsgrammatische Zugänge $\mathrm{zu}$ Phraseoschablonen (und anderen Konstruktionen) unterscheiden sich von phraseologischen Ansätzen wohl am deutlichsten darin, dass sie die sprachlichen Realisierungsbedingungen einer Konstruktion möglichst präzise zu erfassen versuchen. Diese Realisierungsbedingungen können ganz unterschiedlicher Art sein, sie können semantischer, syntaktischer, phonologischer oder pragmatischer einschließlich diskursfunktionaler - Natur sein. Sie können genauso gut die kontextuelle Einbettung (etwa innerhalb einer Periphrase, einer Textsorte oder, in der mündlichen Kommunikation, einer sequentiellen Struktur) betreffen wie die (Flexibilität der) Füllung der Leerstellen selbst. Zur Illustration: Die Reduplikationsstruktur mit an weist keine Constraints hinsichtlich ihrer Einbettungsstrukturen auf, sehr wohl aber hinsichtlich der LeerstellenFüllung: Lizenziert sind allein artikellose Nomen (cf. Tisch an Tisch vs. *der Tisch an dem Tisch) ${ }^{3}$, und es liegt eine semantische Beschränkung auf Konkreta vor (cf. Stein an Stein vs. *Gerechtigkeit an Gerechtigkeit).

Produktivität. Phraseoschablonen (und alle anderen Konstruktionen mit Leerstellen) können unterschiedlich produktiv sein. Die Produktivität einer Konstruktion betrifft die Variation der Leerstellenfüllung, also die Menge an unterschiedlichen Fillern (Instanzen), die in einer Konstruktion realisiert werden können. Die Produktivität einer Konstruktion erhöht sich in dem Maße, wie die Anzahl an unterschiedlichen Instanzen steigt, die die Leerstellen füllen. Ist beispielsweise in einer Reduplikationsstruktur die Menge der potentiell reduplizierbaren Elemente sehr groß - wie etwa bei $\left[\mathrm{NP}_{1}\right.$ an $\left.\mathrm{NP}_{1}\right]$, insofern hier eine große Vielzahl an unterschiedlichen Nomen die Leerstellen besetzen können -, so handelt es sich um eine produktive Konstruktion. Umgekehrt gilt eine Konstruktion als maximal unproduktiv, wenn es nur eine einzige Instanz (etwa nur ein Nomen) gibt, die die Leerstelle spezifizieren kann. Zwischen Constraints der Leerstellen-Füllung und der Produktivität einer Konstruktion besteht mithin ein direkter Zusammenhang. Ähnliches gilt, wie Barðdal feststellt, auch für den Grad an Schematizität und den Grad an Produktivität einer Konstruktion: „The higher the degree of schematicity, the more productive the construction is, and, conversely, the lower the degree of schematicity, the less productive the construction is.“ (Barðdal 2008: 45)

Formale und semantische (Vererbungs-)Beziehungen. Schließlich interessiert sich eine konstruktionsgrammatische Untersuchung von Phraseoschablonen für form- und inhaltsseitige Beziehungen, die eine Ziel-Konstruktion zu benachbarten Konstruktionen unterhält. Der Lokus einer Konstruktion im Konstruktikon, d. h. der Ort im Netzwerk miteinander korrelierter Konstruktionen, ist nicht nur deshalb von besonderem Interesse, weil jede Konstruktion ihren „Wert“ (im Sinne von Saussures „valeur“) erst durch die Einbettung im Relationsgefüge verwandter sprachlicher Einheiten erhält; noch wichtiger ist, dass jede Konstruktion

\footnotetext{
${ }^{3}$ Die Verwendung des unbestimmten Artikels führt zu einer Tilgung der konstruktionsspezifisch-kontinuativen Lesart, so dass im Ergebnis eine andere Konstruktion realisiert wird, cf. ein Tisch an einem Tisch mit der Bedeutung ,ein Tisch angrenzend an einem anderen`.
}

ISSN 1615-3014 
Informationen von hierarchiehöheren Konstruktionen erbt und selbst Informationen an hierarchieniedrigere Konstruktionen vererbt. So erbt die Reduplikationskonstruktion $\left[\mathrm{NP}_{1}\right.$ an $\mathrm{NP}_{1}$ ] formseitig etwa Informationen von binominalen NP-Konstruktionen (cf. Kim/Sells 2015), während ihre Standardlesart (realisiert in Auto an Auto, Stein an Stein usw.) in semantischer Hinsicht Konstruktionen der Quantifizierung nahesteht. Im Einzelfall sind die Beziehungen empirisch genau auszuweisen und zu modellieren, wobei je nach konstruktionsgrammatischem Ansatz die Modellierung durchaus unterschiedlich ausfallen kann (cf. den Überblick in Ziem 2014).

\section{Forschungsschwerpunkte konstruktionsgrammatisch orientierter Analysen von Phrasemen}

Die Konstruktionsgrammatik und die Phraseologie teilen nicht nur ihr Interesse an einem gemeinsamen Gegenstandsbereich, sie weisen darüber hinaus durchaus auch gemeinsame Schwerpunkte bei der Erforschung dieses Gegenstandsbereiches auf. Diese sollen in gebotener Kürze vorgestellt werden. ${ }^{4}$

Grade an Idiomatizität. Die konstruktionsgrammatischen Arbeiten im Rahmen der Phraseologieforschung sind, zumindest zur Gegenstandssprache Deutsch, noch relativ jung. Dies mag zunächst verwundern, denn Idiomatizität war schon die Triebfeder konstruktionsgrammatischer Studien der ersten Stunde (cf. etwa die Schule machende Studie von Fillmore/Kay/O'Connor 1998). Zwar teilen Phraseme mit Konstruktionen auch die wesentliche Eigenschaft, dass ihre form- und/oder inhaltsseitigen Eigenschaften nicht vorhersagbar sind (was dem Verständnis von Konstruktionen als nicht-kompositionelle FormBedeutungspaare entspricht), umso erstaunlicher ist es aber, dass konstruktionsgrammatische Konzepte in der Phraseologieforschung erst eine breitere Rezeption im Anschluss an die erweiterte Bestimmung von Konstruktionen als verfestigte (,entrenched“) und nicht notwendigerweise idiomatische Einheiten (Goldberg 2006: 5) erfahren haben (cf. hierzu Dobrovol'skij 2011; Staffeldt 2011a,b). Der analytische Nutzen eines konstruktionsgrammatischen Zugangs besteht darin, zwischen Graden an Idiomatizität zu differenzieren und ein für phraseologische Analysezwecke hilfreiches Instrument bereitzustellen.

Kognitive Verfestigung als Frequenzeffekt. Es ist inzwischen ein Allgemeinplatz, dass die Musterhaftigkeit von mehrgliedrigen sprachlichen Einheiten (Konstruktionen) ein Effekt ihres rekurrenten Gebrauchs ist (cf. hierzu Gries 2008; aus phraseologischer Perspektive auch Steyer 2013: Kapitel 2). Es überrascht mithin kaum, dass Aspekte der Verfestigung und ihrer empirischen, (korpus-)analytischen Erfassung ein zentraler Untersuchungsgesichtspunkt konstruktionsgrammatisch orientierter Analysen von Phrasemen ist (cf. Stathi 2011; Ziem im Druck). Bei festen Mehrworteinheiten handelt es sich um im Sprachgebrauch stabilisierte sprachliche Muster. Der Verfestigungsgrad seiner möglichen Füllelemente lässt sich unter konstruktionsgrammatischen Vorzeichen etwa mithilfe von Kollokations- und Distributionsanalysen ermitteln (cf. Gries/Stefanowitsch 2004). Entsprechend gilt frequentes Auftreten von sprachlichen Einheiten und Strukturen nicht nur als Indikator für ihre

\footnotetext{
${ }^{4}$ Cf. hierzu auch die Überblicksdarstellung in Ziem/Lasch 2014, insbesondere Kapitel 10, an der ich mich im Folgenden orientiere.
} 
Musterhaftigkeit, sondern auch für einen hohen Grad an kognitiver Verfestigung (,entrenchment“). Phraseologismen und idiomatische Ausdrücke dienen somit einerseits dazu, die konstruktionsgrammatische Theoriebildung zu motivieren (cf. Staffeldt 2011b: 129), andererseits werden sie zunehmend selbst zum Gegenstand konstruktionsgrammatischer Studien.

Musterhaftigkeit und Schematizität. Ein weiterer gemeinsamer Forschungsschwerpunkt betrifft die Musterhaftigkeit komplexer sprachlichen Einheiten selbst. Es waren zunächst insbesondere die Arbeiten von Feilke (1994, 1996), die Möglichkeiten und Grenzen pragmatisch orientierter Analysen von so genannten Ausdruckstypen austariert haben; die Affinität und Nähe zu konstruktionsgrammatischen Untersuchungen ist hier unverkennbar (cf. hierzu auch Dobrovol'skij 2011; Finkbeiner 2008, 2017; Staffeldt/Ziem 2008; Staffeldt 2010, 2011a, b, Ziem/Staffeldt 2011; am Beispiel von Funktionsverbgefüge auch Rostila 2011). Wesentlich ist dabei, dass von der Musterhaftigkeit auf der Ausdrucksebene ausgegangen wird, so dass Ausdrucksprägungen als Ausgangspunkt zur Bestimmung der Flexibilität sprachlicher Einheiten dienen. Zugleich zeichnen sich die Muster durch Schematizität aus: „their slots can be filled by different items“" (Taylor 2004: 224; cf. Finkbeiner 2008: 392). Beispielsweise geht Finkbeiner (2008) Hinweisen auf den konstruktionalen Status von hochfrequenten Idiomen (wie sich etwas an den Hut stecken können, sich etwas in die Haare schmieren können) nach; sie lassen sich einem „Konstruktionsmuster“ zuordnen, das Finkbeiner als [Das kannst du + INFP] angibt, wobei INFP für Infinitivphrase steht. Wie hier exemplarisch deutlich wird, bilden Musterhaftigkeit und Schematizität sowohl für phraseologische als auch für konstruktionsgrammatische Ansätze einen wichtigen konzeptionellen Anker und Orientierungspunkt.

Stabilität und Variabilität. Wie bereits angedeutet (cf. Abschnitt 2), spielen in phraseologischen Untersuchungen von Phrasemen die Untersuchungsparameter Stabilität / Variabilität solange keine herausgehobene Rolle, wie der Fokus auf nicht-schematischen, also lexikalisch voll spezifizierten Mehrworteinheiten und solchen mit externen Leerstellen liegt. Sobald auch Phraseoschablonen einbezogen werden, ändert sich dies. Dobrovol'skijs (2011) grundlagentheoretischer Beitrag zu Phraseoschablonen, von ihm auch „PhrasemKonstruktionen“ genannt (Dobrovol'skij 2011: 111-114), macht deutlich, dass diese zwar als Ganze jeweils eine quasi-lexikalische Bedeutung haben, jedoch Positionen in ihrer syntaktischen Struktur lexikalisch variabel besetzt werden können und dabei bestimmten semantischen (und ggf. syntaktischen) Restriktionen unterliegen, die eines konstruktionsgrammatischen Zugriffs bedürfen (cf. auch Bücker 2012; Dobrovol'skij/Šarandin 2010; Staffeldt 2018; Mellado/Mollica/Schafroth im Druck; Ziem im Druck). Der Grad der Stabilität von Phrasem-Konstruktionen variiert also. Die jeweils konstruktionsspezifische Ermittlung der Variabilität rückt zunehmend in den Fokus der Forschung. Sie ist wichtig, weil sie die Bedingungen des lizenzierten Gebrauchs von Phrasemen betrifft. 
4 Construction Grammar meets Phraseology:

Ausrichtung und Inhalt des vorliegenden Themenheftes

Manche Geburten sind unvorhersehbar schwer, einige erfolgen zu früh, andere nach dem errechneten Termin. Der errechnete Termin für die Publikation des vorliegenden Themenheftes ist lange vorbei. Das Themenheft gehört zu den schweren Spätgeburten (ob das Ergebnis dadurch reifer geworden ist, müssen allein die geneigten LeserInnen entscheiden). Dass es Komplikationen gab, hat in diesem konkreten Fall viele kleine und einige gewichtige Gründe. Umso erfreulicher ist es, dass sich, zumindest aus meiner Sicht, das Warten und Ausharren gelohnt hat - den widrigen Umständen zum Trotz. Ich danke allen geduldigen Beteiligten (die Betroffenen wissen, wer adressiert ist), die diesem Projekt trotz seiner langen Dauer bis zum Ende treu geblieben sind.

Das vorliegende Themenheft umfasst neben dieser Einleitung fünf Fachbeiträge. Jeder Beitrag des Themenheftes liefert eine eigene Perspektive auf das Verhältnis zwischen Konstruktionsgrammatik und Phraseologie. Jeder Beitrag steuert, an konkreten Beispielanalysen illustriert, eine Fülle an Aspekten zur Verhältnisbestimmung bei. Im Folgenden sollen diese Aspekte, gleichsam aus der Vogelperspektive betrachtet, systematisiert werden. Die Liste der erwähnten Aspekte ist freilich nur ein Ausschnitt aus der Agenda, an der sich sowohl phraseologisch als auch konstruktionsgrammatisch interessierte LinguistInnen orientieren können. Abschließend sollen die einzelnen Beiträge vorgestellt und inhaltlich in das Konzept des vorliegenden Themenheftes eingeordnet werden.

Der gemeinsame Ausgangspunkt aller Beiträge des Themenheftes - manchmal explizit, manchmal implizit - bildet die fundamentale Annahme, dass es sich bei Phrasemen und schematischen, grammatischen Konstruktionen nicht um verschiedene Einheiten des Sprachsystems handelt, sondern dass zwischen ihnen vielmehr ein Kontinuum besteht, das eine Bandbreite von Phänomenen im Übergangsbereich von semantisch intransparenten und/oder syntaktisch irregulären festen Mehrworteinheiten (cf. Langlotz in diesem Band) einerseits und grammatischen (Argumentstruktur-)Konstruktionen (cf. de Knop/Mollica in diesem Band; Dalmas/Gautier in diesem Band) andererseits abdeckt. Diesen Übergangsbereich gleichsam sichtbar zu machen und zu vermessen, ist eine Aufgabe des Themenheftes. Dies geschieht einmal aus der Perspektive des phraseologischen Pols des Kontinuums (Langlotz in diesem Band, Filatkina in diesem Band) und einmal aus der Perspektive des konstruktionsgrammatischen Pols (de Knop/Mollica in diesem Band, Dalmas/Gautier in diesem Band). Staffeldt (in diesem Band) nimmt in seiner Studie zur Zweiworteinheit ja aber beide Perspektiven ein. Insgesamt werden dabei folgende thematische Aspekte behandelt:

- Diachron-kontrastive Variation von Konstruktionen, insbesondere Pfade, Vererbungen, Motivationen (Filatkina)

- Kontrastiv-sprachvergleichende Variation von Phrasem- und ArgumentstrukturKonstruktionen (Langlotz, de Knop/Mollica)

- Domänengebunde Variation von Phrasem-Konstruktionen (Langlotz, hier am Beispiel von Emotionen)

- Medialitäts- und registerbezogene Variation von Konstruktionen (Staffeldt; auch: Filatkina) 
- (Nicht-)Kompositionalität von Konstruktionen (Staffeldt; auch: de Knop/Mollica, Dalmas/Gautier, Filatkina)

- Grade von Festigkeit und Dynamik (Staffeldt; auch: Filatkina, de Knop/Mollica, Dalmas/Gautier)

- Semantik/Pragmatik-Syntax-Verhältnis (Dalmas/Gautier; auch: de Knop/Mollica, Staffeldt)

- Kontextuelle / sequentielle Einbettung von Konstruktionen (Staffeldt)

- Verhältnisbestimmung von Konstruktionen, sprachlichen Routinen und formelhaften Wendungen (Filatkina)

- Ausprägungen und Interaktionen von Argumentstruktur-Konstruktionen (Dalmas/Gautier, de Knop/Mollica, hier am Beispiel von Kausal- und Resultativkonstruktionen)

- Interdisziplinäre Relevanz von Konstruktionen, insbesondere für die Psychologie und Cognitive Science (Langlotz; auch: Dalmas/Gautier)

Zunächst widmen sich Sabine de Knop und Fabio Mollica in ihrem Beitrag „Kausalkonstruktionen mit Adjektiv im freien und fixierten Gebrauch“ einer verwandten Spielart der fürs Englische bereits differenziert beschriebenen Resultativ- und caused-motionKonstruktion (cf. etwa Goldberg 1995: Kapitel 7 und 8). Ausgehend von Beispielen wie Ihre Hände waren blau vor Kälte interessieren sich de Knop und Mollica konkret für die Variabilität und Produktivität des Adjektivslots (hier: blau), der das Resultat bzw. das effizierte Objekt (das Hervorgebrachte) des kausalen Vorgangs kodiert. Die Variabilität wird dabei sprachvergleichend (Deutsch, Italienisch, Französisch) ermittelt und dokumentiert. Abhängig vom instantiierten Adjektiv (und Verb) stoßen de Knop und Mollica in ihrer Korpusstudie auf eine Vielzahl an Metaphern und Metonymien. Diese lexikalisch teilspezifizierten Kausalkonstruktionen teilen mit Phrasemen wesentliche Eigenschaften, gelten gemeinhin aber nicht als (prototypische) Vertreterinnen. Ihr phraseologischer Status wird im Übergangsbereich von Phrasemen und Konstruktionen ausführlich diskutiert. Ein besonderes Augenmerk liegt dabei auf der wichtigen Frage, wie frei bzw. wie fixiert Adjektive in den Kausalkonstruktionen tatsächlich sind.

Andreas Langlotz' Studie „Emotions in the face: biology or culture? - Using idiomatic constructions as indirect evidence to inform a psychological research controversy" richtet sich auf phraseologische Konstruktionen mit einer Körperteilbezeichnung als Konstituente (z. B. große Augen machen, die Nase rümpfen usw.). Solche in der Fachliteratur breit diskutierten Somatismen scheinen (teilweise) bedeutungsseitig motiviert zu sein, wenngleich sprachvergleichend eine gewisse Varianz festzustellen ist. Langlotz geht - ebenfalls auf der Grundlage von systematisch erhobenen und ausgewerteten Korpusdaten, erweitert um Befragungen von Muttersprachlern - der Frage nach, inwiefern Emotionen in unterschiedlichen Sprachen durch Somatismen, die sich auf Gesichtsausdrücke beziehen, ähnlich kodiert sind. Langlotz beschränkt sich dabei aus gutem Grund nicht auf typologisch eng verwandte Sprachen, sondern bezieht neben europäischen (Englisch, Deutsch, Französisch, Spanisch) auch ostasiatische Sprachen (Japanisch, Koreanisch und Kantonesisch) ein. Sein Erkenntnisinteresse betrifft nämlich auch die in der Psychologie immer noch kontrovers 
diskutierte Streitfrage, ob Emotionen (wie Freude, Ärger, Zorn, Überraschung) in Gesichtsausdrücken kulturübergreifend gleich kodiert werden. Langlotz zeigt, dass phraseologische Konstruktionen Emotionen in Gesichtsausdrücken sprachübergreifend durchaus ähnlich kodieren, es jedoch ebenso einen beträchtlichen Bereich der kultur- und sprachabhängigen Variation gibt.

Die Studie von Sven Staffeldt „Ja aber - Korpusbasierte Beobachtungen zu einer pragmatisch beschreibbaren Konstruktion“ untersucht ein auf den ersten Blick völlig unscheinbares Phänomen: die Wortkombination Ja aber im mündlichen Sprachgebrauch. Staffeldt zeigt, dass sich mit einem konstruktionsgrammatischen Zugriff neue Erkenntnisse erzielen lassen, die insbesondere pragmatische Aspekte wie kommunikative Funktionen, aber auch ganz grundsätzliche Untersuchungsgesichtspunkte wie (Nicht-)Kompositionalität sowie Verfestigungs- und Stabilitätsgrade von Konstruktionen betreffen. Staffeldt argumentiert, dass die Bedeutungsbestimmung der zweigliedrigen ja-aber-Konstruktion nicht zu trennen ist von der syntaktischen Integration der Einheit einerseits und der Negation der aber-Konstituente andererseits. Die Pragmatik und Semantik der ja-aber-Konstruktion erweisen sich als unentwirrbar miteinander verbunden.

Natalia Filatkina nimmt in ihrem Beitrag „Historische formelhafte Wendungen als Konstruktionen: Möglichkeiten und Grenzen der diachronen Konstruktionsgrammatik“ eine dezidiert diachrone Perspektive ein und argumentiert am Beispiel von formelhaften Wendungen - also mehr oder weniger festen Mehrworteinheiten (oftmals mit pragmatischer Funktion) -, dass sich phraseologische und konstruktionsgrammatische Studien komplementär ergänzen können und vielleicht sogar müssen. Zur Illustration analysiert sie unter anderem formelhafte Wendungen (z. B. mit Verben des Sagens), deren Verfestigungs- und Stabilitätsgrad in semantischer und syntaktischer Hinsicht gegenwartssprachlich hoch ist; aus diachroner Perspektive lässt sich der Prozess der Verfestigung und der (Einschränkung der) Variation motivieren und beleuchten.

Schließlich beleuchten Martine Dalmas und Laurent Gautier in ihrer Untersuchung mit dem Titel „Zur idiomatischen Komponente auffälliger kausativer Konstruktionen im Deutschen“ - ähnlich wie de Knop und Mollica - schematischere Konstruktionen, die in der Regel dem phraseologischen Blick entgehen und auch in der konstruktionsgrammatischen Forschung bislang kaum Beachtung gefunden haben. Anders als Filatkina und Langlotz wenden sie sich also im Konstruktion-Phrasem-Kontinuum stärker dem konstruktionsgrammatischen Pol zu, der sich jedoch durchaus auch aus phraseologischer Sicht als interessant erweist. Konkret zeigen Dalmas und Gautier, inwiefern caused-motion-Konstruktionen (wie jdn. aus dem Bett klingeln) die Phraseologieforschung vor Herausforderungen stellt und wie diese am besten unter Einbezug von konstruktionsgrammatischen Konzepten gemeistert werden können. Zu berücksichtigen seien unter anderem lexikalische Restriktionen bei der Leerstellenfüllung und Unterschiede in der Produktivität von Konstruktionen. Auf der Basis der diskutierten Belege plädieren Dalmas und Gautier dafür, zumindest einige der untersuchten Konstruktionen als phraseologische Einheiten zu verstehen, die es in das ,phraseologische System“ einer Sprache zu integrieren gelte. 


\section{Literatur}

Barðdal, Jóhanna (2008): Productivity. Evidence from Case and Argument Structure in Icelandic. Amsterdam: Benjamins.

Booij, Geert (2002): „Constructional Idioms, Morphology, and the Dutch Lexicon”. In: Journal of Germanic Linguistics 14: 301-329.

Bubenhofer, Noah (2009): Sprachgebrauchsmuster. Korpuslinguistik als Methode der Diskursund Kulturanalyse. Berlin/New York: de Gruyter. (=Sprache und Wissen 4)

Bücker, Jörg (2012): Sprachhandeln und Sprachwissen. Grammatische Konstruktionen in der kommunikativen Praxis. Berlin: de Gruyter.

Colleman, Timothy/de Clerck, Benrad (2011): „Constructional semantics on the move: On semantic specialization in the English double object construction". In: Cognitive Linguistics 22/1: 183-209.

Dalmas, Martine/Gautier, Laurent (2013) : « Les constructions causatives avec mouvement en allemand: d'une saisie phraséologique à une explication constructionnelle ». In : Langages 189/1: 81-213.

Dalmas, Martine/Gautier, Laurent (2018): „Zur idiomatischen Komponente auffälliger kausativer Konstruktionen im Deutschen“. In: Linguistik online 90/3: 145-162.

De Knop, Sabine/Mollica, Fabio (2018): „Kausalkonstruktionen mit Adjektiv im freien und fixierten Gebrauch eine kognitive und konstruktionsgrammatische Untersuchung“. In: Linguistik online 90/3: 21-46.

Dobrovol'skij, Dmitrij/Šarandin, Artëm (2010): „Konstruktionsgrammatik und Lexikographie: Verben der Fortbewegung im ,Neuen deutsch-russischen Großwörterbuch““. In: Durco, Peter (eds.): Feste Wortverbindungen und Lexikographie. Kolloquium zur Lexikographie und Wörterbuchforschung. Berlin/Boston, de Gruyter: 37-41.

Dobrovol'skij, Dmitrij (2010): „Deiktische Konstruktionen des Deutschen aus lexikographischer Perspektive“. In: Dykstra, Anne/Schoonheim, Tanneke (eds.): Proceedings of the XIV Euralex International Congress, Leeuwarden, 6-10 July 2010. CD-ROM. Leeuwarden, Fryske Akademy: 1282-1290.

Dobrovol'skij, Dmitrij (2011): „Phraseologie und Konstruktionsgrammatik“. In: Lasch, Alexander/Ziem, Alexander (eds.): Konstruktionsgrammatik III: Aktuelle Fragen und Lösungsansätze. Tübingen, Stauffenburg: 111-130.

Feilke, Helmuth/Lehnen, Katrin (eds.) (2012): Schreib- und Textroutinen Theorie, Erwerb und didaktisch-mediale Modellierung. Frankfurt a. M. etc.: Lang. (= Forum Angewandte Linguistik 52)

Feilke, Helmuth (1994): Common-sense-Kompetenz. Überlegungen zu einer Theorie des ,sympathischen ' und, natürlichen 'Meinens und Verstehens. Frankfurt a. M.: Suhrkamp.

Feilke, Helmuth (1996): Sprache als soziale Gestalt. Ausdruck, Prägung und die Ordnung der sprachlichen Typik. Frankfurt a. M.: Suhrkamp.

Feilke, Helmuth (2007): „Syntaktische Aspekte der Phraseologie III : Construction Grammar und verwandte Ansätze“. In: Burger, Harald et al. (eds.): Phraseologie. ein internationales Handbuch zeitgenössischer Forschung. Berlin/New York, de Gruyter: 63-76.

Fillmore, Charles J. (1988): “The Mechanisms of ,Construction Grammar”. In: Proceedings of the Fourteenth Annual Meeting of the Berkeley Linguistics Society: 35-55. 
Finkbeiner, Rita (2008): „Zur Produktivität idiomatischer Konstruktionsmuster. Interpretierbarkeit und Produzierbarkeit idiomatischer Sätze im Test". In: Linguistische Berichte 216: 391-430.

Finkbeiner, Rita (2017): “Argumente hin, Argumente her. Regularity and idiomaticity in German N hin, N her”. In: Journal of Germanic Linguistics 29/3: 205-258.

Filatkina, Natalia (2018): „Historische formelhafte Wendungen als Konstruktionen: Möglichkeiten und Grenzen der diachronen Konstruktionsgrammatik“. In: Linguistik online 90/3: 115-144.

Fleischer, Wolfgang (1997): Phraseologie der deutschen Gegenwartssprache. Tübingen: Niemeyer.

Fillmore, Charles J./Kay, Paul/O'Connor, Mary Catherine (1988): "Regularity and idiomaticity in grammatical constructions: the case of let alone". In: Language 64/3: 501-538.

Fried, Mirjam/Östman, Jan-Ola (2004): "Construction Grammar: A thumbnail sketch." In: Mirjam Fried/Jan-Ola Östman (eds.): Construction Grammar in a Cross-Language Perspective. Amsterdam/Philadelphia, Benjamins:11-86.

Goldberg, Adele (1995): Constructions. A Construction Grammar Approach to Argument Structure. Chicago etc.: University of Chicago Press.

Goldberg, Adele (2006): Constructions at Work. The Nature of Generalization in Language. Oxford: Oxford University Press.

Gries, Stefan (2008): "Phraseology and linguistic theory: a brief survey". In Granger, Sylviane/Meunier, Fanny (eds.): Phraseology: an interdisciplinary perspective. Amsterdam/Philadelphia, Benjamins: 3-25.

Gries, Stefan/Stefanowitsch, Anatol (2004): "Extending collostructional analysis. A corpusased perspective on 'alternations"'. In: International Journal of Corpus Linguistics 9/1: 97-129.

Hoffmann, Thomas/Graeme, Trousdale (eds.) (2013): The Oxford Handbook of Construction Grammar. Oxford, Oxford University Press.

Jackendoff, Ray (2008): "Construction after construction and its theoretical challenges". In: Language 84/1: 8-28.

Kay, Paul/Fillmore, Charles F. (1999): "Grammatical constructions and linguistic generalizations: The 'what's x doing y?' construction”. In: Language 75/1: 1-33.

Kay, Paul (2002): “An informal sketch of a formal architecture for construction grammar”. In: Grammars 5: 1-19.

Kim, Jong-Bok/Sells, Peter (2015): "English binominal NPs: A construction-based. perspective. In: Journal of Linguistics 51: 41-73.

Langlotz, Andreas (2018): "Emotions in the face: biology or culture? - Using idiomatic constructions as indirect evidence to inform a psychological research controversy". In: Linguistik online 90/3: 47-74.

Mellado Blanco, Carmen/Mollica, Fabio/Schafroth, Elmar (im Druck): „Phrasemkonstruktionen aus kontrastiver Sicht: eine korpusgestützte Analyse zum Deutschen, Italienischen und Spanischen“. In: dies. (ed.): Konstruktionen zwischen Lexikon und Grammatik. Phrasemkonstruktionen im Deutschen, Italienischen und Spanischen. Berlin/Boston: de Gruyter. 
Michaelis, Laura A. (2012): "Making the Case for Construction Grammar". In: Boas, Hans C./Sag, Ivan (eds.): Sign-Based Construction Grammar. Stanford, CSLI Publications: 31-69.

Östman, Jan Ola (2004): "Construction Discourse: A prolegomenon". In: Östman, JanOla/Fried, Mirjam (eds.): Construction grammars: cognitive grounding and theoretical extensions. Amsterdam, Benjamins: 121-144.

Rostila, Jounis (2011): „Phraseologie und Konstruktionsgrammatik. Konstruktionsansätze zu präpositionalen Funktionsverbgefügen“. In: Prinz, Michael/Richter-Vapaatalo, Ulrike (eds.): Idiome, Konstruktionen, „verblümte rede“. Beiträge zur Geschichte der germanistischen Phraseologieforschung. Stuttgart, Hirzel: 263-282.

Staffeldt, Sven (2010): „Zur Rolle des Körpers in der phraseologisch gebundenen Sprache. Fingerübungen zur semantischen Teilbarkeit“. In: Korhonen, Jarmo et al. (eds.). EUROPHRAS 2008. Beiträge zur internationalen Phraseologiekonferenz vom 13.-16.8.2008 in Helsinki.

Staffeldt, Sven (2011a): „,Wie Sie wissen“. Kleines Plädoyer für mehr Pragmatik und mehr Konstruktionen in Grammatiken“. In: Sprachwissenschaft 36: 85-112.

Staffeldt, Sven (2011b): „In der Hand von Konstruktionen. Eine Fallstudie zu bestimmten Phraseologismen mit in ... Hand“". In: Lasch, Alexander Lasch/Ziem, Alexander (eds.): Konstruktionsgrammatik III: Aktuelle Fragen und Lösungsansätze. Tübingen, Stauffenburg: 131-147.

Staffeldt, Sven (2018): Gebrauchssemantik von Hand. Korpusbasierte Studien zu somatischen Phraseologismen des Deutschen mit der Konstituente Hand. Tübingen: Stauffenburg.

Staffeldt, Sven (2018): „Ja aber - Korpusbasierte Beobachtungen zu einer pragmatisch beschreibbaren Konstruktion“. In: Linguistik online 90/3: 75-114.

Staffeldt, Sven/Ziem, Alexander (2008): „Körper-Sprache: Zur Motiviertheit von Phraseologismen mit Körperteilbezeichnungen“. In: Sprachwissenschaft 33/4: 455-499.

Steyer, Kathrin (2013): Usuelle Wortverbindungen. Zentrale Muster des Sprachgebrauchs aus korpusanalytischer Sicht. Tübingen: Narr.

Stathi, Katerina (2011): „Idiome in der Konstruktionsgrammatik: Im Spannungsfeld zwischen Lexikon und Grammatik". In: Lasch, Alexander/Ziem, Alexander (eds.): Konstruktionsgrammatik III. Aktuelle Fragen und Lösungsansätze. Tübingen, Stauffenburg: 149-163.

Taylor, John R. (2002): Cognitive Grammar. Oxford: Oxford University Press.

Taylor, John R (2004): Linguistic Categorization. Prototypes in Linguistic Theory. 3. Aufl., Oxford: Oxford University Press.

Taylor, John (2012): The mental corpus. How language is represented in the mind. Oxford: Oxford University Press.

Ziem, Alexander/Boas, Hans C. (2017): "Towards a Constructicon for German". In: Proceedings of the AAAI 2017 Spring Symposium on Computational Construction Grammar and Natural Language Understanding. Technical Report SS-17-02: 274-277.

Ziem, Alexander/Lasch, Alexander (2013): Konstruktionsgrammatik. Konzepte und Grundlagen gebrauchsbasierter Ansätze. Berlin/Boston: de Gruyter.

Ziem, Alexander (2014): „Konstruktionsgrammatische Konzepte eines Konstruktikons“. In: Lasch, Alexander/Ziem, Alexander (eds.): Grammatik als Netzwerk von Konstruktionen. 
Sprachliches Wissen im Fokus der Konstruktionsgrammatik. Berlin/New York, de Gruyter: 15-34.

Ziem, Alexander/Staffeldt, Sven (2011): "Compositional and embodied meanings of somatisms: a corpus-based approach to phraseologisms". In: Schönefeld, Doris (ed.): Converging evidence: Methodological and theoretical issues for linguistic research. Amsterdam/Philadelphia, Benjamins: 195-219.

Ziem, Alexander (2008): Frames und sprachliches Wissen. Kognitive Aspekte der semantischen Kompetenz. Berlin/New York: de Gruyter. (= Sprache und Wissen 2)

Ziem, Alexander (im Druck): „Tag für Tag Arbeit über Arbeit: Konstruktionsgrammatische Zugänge zu Reduplikationsstrukturen im Deutschen“. In: Steyer, Kathrin (ed.): Sprachliche Verfestigung. Wortverbindungen, Muster, Phrasem-Konstruktionen. Tübingen: Narr. (= Studien zur Deutschen Sprache 79) 